\title{
A Community of Knowledge Management Practitioners: Mirroring Power across Social Worlds
}

\author{
Hiroko Wilensky, Norman Makoto Su, David Redmiles, and Gloria Mark \\ University of California, Irvine, USA, \{hwilensk, normsu, redmiles, gmark\}@uci.edu
}

\begin{abstract}
In our previous study, we focused on two spaces: a community of knowledge management (KM) practitioners and their respective work organizations. We found that the "community" largely existed to legitimize KM practices, rather than to learn KM practices. Our current study builds upon this work by uncovering how in fact power relationships in work organizations are transferred and mirrored into the community. Consequently, these relationships shape and define the community's processes: they set up boundaries of the community, reinforce the knowledge sharing practices among the members, and institutionalize community members' beliefs of KM. We have adopted Strauss's social world perspective to better understand how the actions and interactions outside of the community impact and mold the community.
\end{abstract}

Keywords: Knowledge management, Social worlds, Community of practice, Power, Aerospace industry

\section{Introduction}

For the past three years, we have been conducting an ethnographic investigation into a forum for knowledge management practitioners (KMPs) from the aerospace industry. According to their official website, this forum is a locale where "leaders in knowledge management in industry (with a focus on aerospace industry) and academia come together to share, collaborate, and discuss." Coming from five different aerospace organizations, these KMPs participate in a quarterly face-toface meeting called the "Mid West Aerospace Industry KM Exchange Community of Practice" " (hereafter abbreviated as the KM Exchange).

Our previous study (Su et al., 2007) revealed that a community (one labeled as a "community of practice") can have motivations that stem beyond the cultivation of an environment for mutual and reciprocal learning and supporting. Instead, the

\footnotetext{
${ }^{1}$ All names and locations in this paper have been anonymized.
} 
KM Exchange was found to be a crucial legitimizing and affirming conduit for practitioners' KM practices in their respective work organizations. The KM Exchange served as a pulpit of sorts for its senior members to expound the worthiness of $\mathrm{KM}$ as a discipline of priority.

In this paper, we further explore the relationships among the members; in particular, we examine the role that power plays. We question how power relationships have influenced the boundaries of the community, knowledge sharing among community members and institutionalized beliefs of KM. We argue that power relations which exist in one's respective communities can be transferred and mirrored in new cross-organizational communities. In our analysis, we turn to the notion of social worlds explicated by Strauss (1978) as a way to understand how this community, despite its mantra of equalizing or flattening traditional hierarchical structures, is nevertheless influenced by the activities and experiences of the members in their work organizations as well as other interactions outside of the community, such as attending conferences and collaborating on KM projects.

Although members of the KM Exchange often call their forum a "community," their community has qualities distinctively different from well-known models of communities. Lave \& Wenger's (1991a) communities of practice (CoP) is an immensely popular conceptual model of community among KM practitioners. This CoP theory posits that the mentor-pupil model is outmoded and stresses instead that learning occurs among peers and cohorts in a participant's periphery. Through legitimate peripheral participation, new members are able to move towards the core of a community, becoming experts or old-timers. KM practitioners view $\mathrm{CoP}$ as an ideal model to emulate in their organization. Countless books provide step-by-step instructions on "creating" CoP (e.g., Rumizen, 2002; Wenger, 2002).

It is our contention that $\mathrm{KM}$ practitioners tend to view a community as an isolated space for learning, knowledge sharing, and networking among its members (Dalkir, 2005). Brown \& Duguid (2000), for example, describe CoP as tight-knit groups of people who work together directly. Lave \& Wenger (1991) touch upon the need to expand the analysis of learning beyond the immediate context, but much of their work has focused squarely on one space and its social dimensions. For example, the analysis of meat cutters and an alcoholic support group primarily focus on the ongoing interactions of a single space.

We instead propose to see the KM Exchange as one of intertwined social worlds in which the members are involved. A social world (Strauss, 1978) is a collective unit of individuals with shared commitments who gather to perform a primary activity. Socials worlds are a highly fluid social structure, which constantly changes due to processes such as conflict, competition, negotiation, and exchange. Importantly, social worlds can intersect with other social worlds under a variety of conditions. Although anyone in a social world is associated with its actions, some members carry the power to decide which members are more "authentic" (see Section 3.2) via which social mechanism. Our analysis has shown 
that individuals who have the power to determine authenticity in work organizations have the power in the KM Exchange. These individuals play a crucial role as spokespersons: Latour (2005) notes that spokespersons "speak for the group existence... all [groups] need some people defining who they are, what they should be, what they have been...justifying the groups' existence." We are attempting to uncover the processes by which spokespersons of organizations can become spokespersons of other organizations, thus reaffirming their appropriate role across multiple social worlds.

In this paper we first describe our field site setting and methodology. We then briefly describe the aerospace industry, followed by a recap of our previous study (Su et al., 2007) on the "rhetoric" of aerospace KMPs in the KM Exchange and in their respective workplaces. The next sections detail our new analysis incorporating the social world perspective. This mindset allows us to unravel the power relations that permeate from the work organizations into the KM Exchange community. Our central idea is that power structures emanating from multiple social worlds can reassemble themselves in new social worlds (or communities), dramatically defining the community's trajectory. This can transpire despite a community's mantra of equal sharing.

\section{Methodology}

Our data collection and analytical methods are steeped in the grounded theory (Strauss, 1998) practice of thematic generation. We carried out both participant observations and one-on-one interviews with members of the Midwest KM Exchange. It is a physical forum where aerospace employees interested in KM meet on a quarterly basis. Other members include people from universities and power companies. The meetings' physical location was rotated among the participants' organizations. The meetings usually lasted at least half a day (4.5 hours on average) and had a standard schedule of: 1) networking, 2) presentations about KM, 3) lunch plus networking, and 4) splitting up into small (roughly 5-10 people) break-out discussion groups concentrating on specific KM topics of interest. Interviewees were recruited at the KM Exchange and through snowball sampling.

This paper builds upon our previous dataset (Su et al., 2007), bringing it up to a total of eight meetings, three conferences, and 23 semi-structured interviews over three years. In this paper, we refer to the four founders of the KM Exchange as the core members. We call senior members who are designated as the representative of their company in the community the focal members. All the core members are also focal members. 


\section{Background: The Aerospace Industry}

Compared to other businesses, the aerospace industry manufactures highly specialized technical components such as satellites, aircraft, and guided missiles. Only a few large companies have the technical competence and resources to create such products. Aerospace companies must seek out a uniquely skilled work force of scientists, engineers, and manufacturing workers. For security purposes, the export of aerospace goods is regulated by government agencies. An increase of global collaborations in the aerospace industry has led to new challenges in data sharing with government oversight (Lorell et al., 2002). As a result, aerospace companies have a culture of secretiveness and protectiveness of their intellectual property. Finally, there is a workforce "crisis" in the aerospace industry due to the loss of jobs from reduced defense budgets after the Cold War. According to the 2006 Aerospace Industries Association of America CEO's statement, the average age of manufacturing employees was 51 and 54 for engineers, with an estimated $27 \%$ of workers becoming eligible in 2008 (AIA, 2006).

\subsection{Knowledge Management Practitioners in Aerospace}

We now briefly summarize our findings about KM practitioners in the aerospace industry (Su et al., 2007). Our study examined the "rhetoric" of KMPs in their organizations and their community (the KM Exchange). By critically looking at how people talk and legitimize KM as a viable discipline in and out of their professional circles, we can describe how KMPs define KM and its usefulness. KMPs emphasize that KM is a way to efficiently manage knowledge: finding "lost" knowledge and eliminating "redundant" knowledge. The aerospace industry's special attributes make KM even more imperative; for example, the aerospace industry often hires people with rare talents (e.g., experts on Martian terrain). Losing such an employee is equated to losing knowledge. KM tools such as expert locators and $\mathrm{KM}$ practices such as $\mathrm{CoP}$ are regarded as progressive tools that are capable of solving knowledge issues. At the same time, KMPs are careful to set themselves apart from information technology (IT) personnel and tools (e.g., databases), noting that knowledge is not information and that knowledge is a far more complex and subtle entity (e.g., by delineating tacit and explicit knowledge). Moreover, KMP are personally equipped to understand the social nature of knowledge, having experience in social and organizational behavior (e.g., noting that software tools cannot be deployed without proper evaluation of a workplace's culture). Finally, KMPs argue that KM is even more appropriate for the aerospace industry in order to combat its secretive, counter-productive culture. $\mathrm{KM}$ is seen as a way to overcome a company environment where employees are mindful of sharing data, even among their peers, hence increasing company effectiveness. 
We also observed KMPs "talking shop" in the KM Exchange. Despite the KM Exchange's own label as a community of practice, we found the community to be less about learning KM skills from peers and elders via legitimate peripheral participation, and more about learning how to "spread" the gospel of KM. In particular, we found the majority of discussions on and off-line to be about KM's legitimacy: people sharing their pains in getting the proper constituents to understand KM's value, how to embed KM throughout work processes at the organization, and how to establish $\mathrm{KM}$ as a reputable discipline. While disciplinary legitimacy was the usual topic at hand, we found that newbies ${ }^{2}$ felt the community was stagnating - they were not learning enough about KM itself. The focal members, the oldies, wanted to promote, while newbies wanted to learn.

This disparity of motivations between the oldies and newbies leads us to the main focus of this paper. $\mathrm{CoP}$ are by far the most popular approach for fostering cross organizational sharing in the $\mathrm{KM}$ field. Books on cultivating or implementing CoP abound (Denning, 2005; Hasanali et al., 2002; Rumizen, 2002; Saint-Onge \& Wallace, 2003; Wenger, 2002). At its heart, KM views CoP as a way to bring disparate groups together to foster sharing, thus, efficiently using knowledge and leading to increased company productivity. While the literature acknowledges that $\mathrm{CoP}$ require time and effort to implement, we feel that it often ignores the social structure inherent in the groups that CoP seek to bring together. Our previous study revealed that attributes from these groups can make so-called CoP very much different from Wenger's ideal. We now draw upon Strauss's social world model to allow us to explicate the community that the KM Exchange represents.

\subsection{The Social World Perspective}

According to Strauss (1978), social worlds have a primary activity and sites where these activities occur. Notably, Strauss describes a social world as a fluid and dynamic entity, often intersecting with other social worlds. Actions and interactions in one social world may significantly impact other social worlds. For example, Mark \& Poltrock (2003) note that technology adoption can transfer from one social world to another: they describe how a virtual meeting system was adopted across different social worlds, changing in its usage (e.g., from being a teaching tool to a document sharing application) and its acceptance (e.g., rejection or acceptance by gatekeepers in social worlds). In discussing the analytical practicality of this theory, Strauss describes several focal points of interests that arise when using a social world's perspective. However, in this paper, we focus on authenticity. Authenticity pertains "to the quality of action, as well as to judgments of which acts are more essential." Intertwined with authenticity is the

2 Newcomers and focal members are used interchangeably with newbies and oldies, respectively. 
issue of power. Those who wield power in the social world can decide which members are authentic. Moreover, those in power decide how newcomers are initiated into the social world. Strauss also stresses the importance of a social world's history. He admonishes those that "focus on contemporary life while either avoiding history or using it as a backdrop for the analysis of ongoing organizations and processes." Indeed, as we will discuss, a social world's history can have dramatic effects on future social worlds. Analogously, a social world's history of power can elucidate current power structures.

We argue that, sometimes, what one ends up doing by combining or bringing together multiple social groups is a sort of mirroring or replication of power structures. In other words, power structures inherent in the social worlds end up being transferred over and reestablished/reinforced in new social worlds.

\section{A Social World Perspective into Power in the KM Exchange}

Building upon our previous work, we now focus our analysis on how power features of social worlds have shaped the KM Exchange. In particular, we see how power relations in social worlds have played a role in 1) the founders, 2) the newcomers, 3) disparity of motivations between "oldies" and "newbies," and 4) delineating KM for members, resulting in the institutionalization of beliefs on KM within the community. The KM Exchange's power is largely concentrated in the core members who authenticate (via social mechanisms) the activities in the KM Exchange.

Social worlds can be in countless discernible forms: temporary or long-lived; small or large; local or international; emergent or established; virtual or physical; and with tight boundaries or permeable boundaries. Through the analysis of our field data, we identified these key social worlds surrounding the KM Exchange: the established aerospace corporations, the local universities, the master's degree KM program which one of the core members helped found, various KM conferences, KM project collaborations among some focal and senior members, and the tight-knit circle of the core members.

\subsection{Founders across the Work Organization \& the KM Exchange}

The core members cemented their power in the KM Exchange by being the progenitors of the community. This establishment of power and leadership in KM in fact is a reflection of their own initiatives in the social worlds of their workplaces. All the core members were responsible for starting the KM team/department or were appointed as the head of KM efforts in their respective work organizations. Two core members brought up their pet knowledge-based projects to their upper management and these projects eventually morphed into a 
larger KM initiative, whereas others were hired by the corporation specifically to spearhead a new KM effort. One core member explained about his company's search for someone who could lead KM: "The committee had certain level of effectiveness and tried to move initiative forward, but there was no single individual who was accountable...um...that could really lead the group and lead the broad group, not so much built an empire of knowledge management, but really coordinate across the company, great diversity of different organizations of the company to get everybody to kind of get move into the same direction effectively." Thus, these core members represented the leadership for establishing and encouraging $\mathrm{KM}$ in their organizations.

A number of small, short-lived social worlds existed prior to the formation of the KM Exchange, and these temporary social worlds eventually led to a tight-knit social world of the core members. The core members founded the KM Exchange after several small encounters at KM-styled conferences and subsequent lunch gatherings. Through these informal get-togethers, the core members formed a tight-knit, if not exclusive, group of KM leaders. Throughout our interviews, the core members described a special bond that existed between them: "It's the four of us <laugh> who constantly bug each other...I think there's a closer connection. Like, I mean, Sam asked [me a KM question] at ten o'clock at night, and I didn't even hesitate to respond."

Despite the short existence of the KM Exchange, when we interviewed our informants, many of them could not accurately recall how the KM Exchange had initially started. Indeed, our informants provided us a number of curious variations on how the KM Exchange came into existence. The interpretations ranged from the simple, assertive answers of "I created it" and "I founded the community" by two core members, to the answer that it was a spin off from a preexisting KM forum in a particular aerospace company in which another core member was in charge of the KM team. These contradictory stories are indicative of the importance the creators of the KM Exchange place on holding power not only in their own organizations, but also in their discipline's cross-organizational community. As forerunners of KM in their own social worlds, the core members seek to reestablish their priority and repute in the KM Exchange as well.

\subsection{Newbie Initiation into the Community}

As we mentioned, the core members seek to establish a new community that nevertheless mirrors their place in the power hierarchy in their respective home organizations. They can continue to assert their power by creating a population of newcomers in the KM Exchange primarily from their own subordinates. Newcomers join the KM Exchange by being invited by the focal members or senior members. The majority of the newcomers usually work for the focal members, with the rest being students who are attending a KM master's degree program at a local university (which one of the core members helped establish; 
this core member is also a newly appointed adjunct faculty in this university). A newcomer explained to us that becoming a KM Exchange member is really an informal job requirement: "Um...when I joined the group, they said, 'Hey, there is a meeting and you are going.' <laughs $>$." Other newcomers told us that they were invited to attend the KM Exchange by the focal members: "I was pretty much asked to go and do it back in 2000"; "The KM staff are invited, but it is optional." Because the focal members are authoritative figures in their work organizations, recruiting the new KM Exchange members was relatively easy for them. Moreover, because these newcomers were subordinates, they often felt obligated to go.

Although we had initially expected the KM Exchange to foster dynamic and frequent interactions as well as help the members establish a vibrant social network, we were surprised to learn that the majority of newcomers and some senior members had little or no interactions with other members once a meeting concluded. One newcomer noted that off-line communication (i.e., not during meetings) among peers was done only through core members: "Because I talked to several people at the meeting and I would like to be able to contact them again... and I normally have to go through someone like Thomas or Ken [core members] to get information." One senior member noted that networking is the focal member's job: 'I don't have the time to be out there building relationships. Ken who is the higher level, that's his responsibility. That's his task to be building the relationships, making the connections, providing that for us. The rest of us-I personally don't do that because I don't have the time I just have too much on my mind." In contrast to the oldies, newbies had little connection with each other. While we observed a very tight-knit social world of the core members, we did not similarly observe a social world of newbies emerging from the KM Exchange: certainly, we observed interactions of newbies at meetings occurring between members of the same work organization, but little between newbies of different organizations. One might conjecture that hobnobbing with the boss carries the same subtle power dynamics as hobnobbing with the boss's own cohorts/peer. The social worlds and their hierarchical structure from which the newbies and oldies come from thus in some sense get replicated into the KM Exchange, possibly hindering legitimate peripheral participation among newbies.

\subsection{Disparity Between the Newbies and Oldies: Stagnation}

While in its nascent stages, membership boundaries of the KM Exchange were a concern: who could become members? During the meetings, boundary issues were often debated upon. Members asked about inviting the local KM master's degree program students which included some international students and KM practitioners from non-aerospace industries - this raised security issues. The debates would always end when some senior members voiced their opinion that the community should remain only within the aerospace industry. One senior 
member said, "Our identity is 'aerospace' KM. Better to focus on 'aerospace." All the core members seemed content with the boundaries of the community: "I' $d$ like to keep it an aerospace focus."

On the other hand, our interviews revealed that newcomers and some senior members had different ideas about the boundaries. Newbies sometimes remarked on the domineering personalities of the core members: "Sometimes, the meetings are dominated by strong characters," or "I worry that we're going to hear from the same people over and over again." These power relations played out in disappointing meeting content for many newbies. One junior KM staff member explained why she didn't attend recent meetings: "Because a lot of them are really repetitive. That was good for the first maybe three or four [meetings] and after a while people just started talking about the same things over and over again. And then...so I was kind of losing interest <laughs> because I'm not learning anything new at these things." Another newbie gave a lukewarm answer about the meeting's usefulness: "I mean, I don't find any meeting extremely helpful. They are all somewhat useful. I haven't found any of them to... if I hadn't attended, you know, my outlook on knowledge management and what I do would be not much different." One senior member informed us: "My opinion is [the KM Exchange is] a little stagnated. You know, we can share so much for so long. I think we need to do...we might venture out and include more people." This sense of stagnation in the community reveals a disparity between those whose desire is to learn KM and those who need to legitimize KM practices to stay alive. In other words, the focal members have dictated the KM Exchange's content, which seems to serve a purpose contradictory to the CoP's supposed benefits.

At the second quarterly meeting in 2007 , the focal members announced that they would make one of the quarterly meetings a conference open to other industries. This idea was already mentioned by one of the core members in September 2006 at our interview; therefore, it is not evident whether this core member reshaped the boundaries of the KM Exchange on his own cognizance or stagnation led to this decision. Nevertheless, the expansion of the KM Exchange serves to strength the core members' place in a reciprocal manner. One focal member commented on the expansion: "They want to grow the group. There are a couple pressures that make them want to grow the group...the general theme for growing the group is that they want knowledge management to be the idea to spread. And at least for the term to be recognized [in their work organizations] more, what I would call legitimizing it." In other words, expansion of the group's boundaries allows the KM Exchange to become more reputable and therefore further legitimize KM in their own organizations.

One core member invited a professor from a local university whom he met at a conference. Because of this professor's enthusiastic and assertive nature and the prestigious nature of his social world (academia), he quickly moved to the center of the KM Exchange. He hosted a quarterly meeting and a conference sponsored by the KM Exchange that featured presenters and participants from other industries (e.g., construction engineering and high-tech) at his university. 


\subsection{The Delineation of KM by the Focal Members}

Although the KM Exchange proclaims that it is a locale for its members to share their knowledge on KM, we found knowledge sharing practices were actually largely shaped by the focal members.

The majority of the members in KM Exchange work for or their work is associated with the focal members. Knowledge of KM was passed down from the focal members to their associates and subordinates at their work places. The focal members would impart a variety of KM tools and techniques via lists of books to read and types of conferences to attend. For instance, one newcomer explained with admiration on how her boss is knowledgeable of KM: "Sam has a library of wonderful knowledge, lots of knowledge, all kind of books...he got, he basically gives us all copies of different books from that, wherever he quoted <laugh> wherever he quoted, anything he finds, anything good books on KM practices, he makes sure we all get that." Another newcomer described how she learned KM at her workplace: "Just listening to Thomas. Every time, he would pull together like an impromptu meeting-I used to go [to] all [of them]-every time he gave a briefing to someone explaining knowledge management and what the knowledge management office is going to do, what do they project for the future, I would attend those...Book[s], he gave me lots of, lots of books to read <laughs $>$. Yeah, there was a lot of that." The focal members essentially defined KM for their subordinates in their organizations.

The focal members also prescribed which KM conferences their members were to attend. For example, the East Coast Knowledge Forum was a conference mentioned by some of the core members in our interviews. Many members also attend APQC conferences. One member described his experience at one of the conferences: "I met people like Larry Prusak and Hubert Saint-Onge at different events. Sam [my boss] had me three months into my knowledge management $<$ laughs $>$ career, sent me to East Coast Knowledge Forum in [city] to represent our company. I was really kind of blown away, but I got to meet the who's who in knowledge management at that particular conference and it was very enlightening."

Not only is the "curriculum" outlined by the focal members in the workplaces, but also in the KM Exchange. The community's meetings are planned exclusively by the focal members. At each meeting, either during lunch or breakout discussions, the focal members gather at a separate exclusive table to discuss future meetings and the future direction of the KM Exchange. In between the quarterly meetings, the focal members also exchange emails with each other in order to determine the schedule and content of the presentation topics. Members were often allowed to vote upon a set of topics they wished to discuss in the final break-out discussions sections closing the KM Exchange sessions.

Accordingly, we observed some beliefs on KM have become institutionalized within the community. It became difficult for newbies to oppose these prevailing beliefs. Often, newbies accept these beliefs without questioning. Two of the most 
common beliefs on KM we heard from our informants and at the quarterly meetings were: 1) the aging workforce issues will cause a serious knowledge drain and 2) the aerospace culture needs to be changed to a sharing culture. From our data, the informants' discussions on these two issues seemed too uniform and scripted for us, leading us to wonder whether this may be the result of the focal members' delineation of the KM Exchange. For example, statements from our data set like "people are retiring left and right...that's going to cause a big hole" by one newbie are typical with regards to the first belief. Contrary to such prevailing accounts, we found varied opinions in other arenas. For instance, AARP (formerly American Association for Retired Persons) recently reported that people often plan to work beyond their retirement age due to various reasons (Brown, 2003). Moreover, at a conference sponsored by the KM Exchange, one presenter from a high-tech company dismissed the aging workforce crisis by questioning the actual value of the technological knowledge that retiring workers have, given the rapidly changing nature of the engineering fields: "Today's technology is different from yesterday's technology." We are not attempting to prove that the focal members' KM beliefs are incorrect here; rather, we believe these counterarguments show that such KM views are contestable, and the little variety in opinions among the KM Exchange members on these views are indicative of the institutionalization of KM beliefs by those in power.

KM "knowledge" and techniques are passed down from the focal members to their associates and subordinates at their work places. In turn, the KM Exchange played a role in reinforcing the power the focal members have in defining and delineating $\mathrm{KM}$ for its members and subordinates. This control over the authenticity - the judgment of the quality of actions - is a key shaper and force for institutionalizing the KM Exchange's content.

\section{Conclusion}

In our previous study, we learned that the KM Exchange exists largely for legitimization rather than for learning. Legitimization of KM practices was crucial for the KM practitioners' survival in their respective work organizations. In our current study, we extended our analysis to focus on the power relationships within the KM Exchange and the social worlds surrounding this forum. We adopted Strauss's notion of social worlds to better understand these power alliances. We analyzed how power has impacted the boundaries of the KM exchange, its knowledge sharing practices, and finally the members' beliefs on KM.

Following Strauss's (1978) call for a historical perspective on social phenomenon, we found it imperative for us to examine the narrative of how past events intersecting multiple social worlds has lead to the formation of the KM Exchange and its current power characteristics. What we found was that the power structures extant in the social worlds of some members become mirrored into new 
social worlds. Being leaders in their own respective work organizations, these same leadership characteristics helped the core members form the KM Exchange. As authoritative figure in their organizations, the focal members brought their subordinates into the KM Exchange, thus reinforcing their authority in the community. Subjected to predefined "courses" of study, new members learned KM primarily from their focal member at their workplaces. This learning practice is then reinstated at the KM Exchange whose content is again largely shaped by the focal members. While new members often look up to the focal members with reverence and admiration (viewing them as authorities, just as they view their own boss as one), they also soon notice that the forum is becoming stagnated, leading to little learning of new KM techniques or skills. We also observed newbies accept without question the beliefs on KM passed down from the focal members. Some beliefs on KM are nearly without variation among the members and quickly become institutionalized within the community. This makes these beliefs difficult for newbies to contest. These events shaped and defined the KM Exchange into its current form, a reflection of discipline spokespersons' power within work organizations.

The hierarchical structure of the KM Exchange is distinctively different from the community of practice model. KM typically view $\mathrm{CoP}$ as a closed, isolated space for learning, knowledge sharing, and networking. KMPs often see CoP as a democratizing entity, leveling out chains of command. Yet, we ascertained that power relationships in and out of the KM Exchange dramatically shaped the community itself and its members' beliefs on KM. We argue that to truly understand a community, one needs to examine the actions and interactions in the social worlds beyond the community and how these social worlds intersect. In particular, when "implementing" $\mathrm{CoP}$, one may end up merely transferring and mirroring extant social worlds, leading to little legitimate peripheral learning, but the strengthening of already existing power relations.

\section{References}

AIA: Statement by Mr. John W. Douglass President \& Chief Executive Officer Aerospace Industries Association of America (2006). Available via www.aiaaerospace.org/aianews/speeches/2006/statement_douglass_061306.pdf. Accessed 28 Mar 2008.

Brown, J.S., and Duguid, P.: The social life of information. Harvard Business School Press, Boston (2003).

Brown, K.: Staying ahead of the curve 2003: The AARP in retirement study (2003). Available via www.aarp.org/research. Accessed 4 Feb 2008.

Dalkir, K.: Knowledge management in theory and practice. Butterworth-Heinemann, Burlington, MA (2005).

Denning, S.: The leader's guide to storytelling: Mastering the art and discipline of business narrative. Jossey-Bass, San Francisco (2005). 
Hasanali, F., Hubert, C., Newhouse, B., O’Dell, C., Westal, W., et al.: Communities of practice: A guide for your journey to knowledge management best practice. American Productivity \& Quality Center, Houston (2002).

Latour, B.: Reassembling the social: An introduction to actor-network-theory. Oxford University Press, New York (2005).

Lave, L. and Wenger, E.: Practice, person, social world. In H. Daniels (Ed.), An introduction to Vygotsky, pp.143- 150. Routledge, New York (1991).

Lave, L. and Wenger, E.: Situated learning: Legitimate peripheral participation. Cambridge University Press, Cambridge, UK (1991a).

Lorell, M.A., Lowell, J., Moore, R.M., Greenfield, V.,Vlachos, K., et al.: Going global? U.S. government policy and the defense aerospace industry. RAND Corporation, Washington D.C. (2002)

Mark, G., and Poltrock, S.: Shaping technology across social worlds: groupware adoption in a distributed organization. Proc. ACM Conf. Supporting Group Work GROUP'03, 284-293 (2003).

Rumizen, M. C.: The complete idiot's guide to knowledge management. Alpha Books, Indianapolis, IN (2002).

Saint-Onge, H., and Wallace, D.: Leveraging communities of practice for strategic advantage. Butterworth-Heinemann, Burlington: MA (2003).

Strauss, A.: A social world perspective. Studies in symbolic interactions, 1(1), 119-128 (1978).

Strauss, A., and Corbin, J.: Basics of qualitative research: techniques and procedures for developing grounded theory. SAGE Publications, Thousand Oaks: CA (1998).

Su, N., Wilensky, H., Redmiles, D., and Mark, G.: The gospel of knowledge management in and out of a professional community. Proc. ACM Conf. Supporting Group Work GROUP' 07, 196-207 (2007).

Wenger, E., McDermott, R., and Snyder, W. M.: Cultivating communities of practice. Harvard Business School Press, Boston (2002). 